\title{
A Comparative study of heart rate variability during Valsalva maneuver in healthy, hypertensive and hypertensive diabetic subjects
}

\author{
Dr. Lata Patil ${ }^{1}$, Dr. Archana Jadhav ${ }^{2}$, Dr. N. G. Borade ${ }^{3}$ \\ 1-Dr.D.Y.Patil Medical college and Research Institute/Dr. D. Y. Patil University/Pune, Maharashtra, India. \\ 2- Dr.D.Y.Patil Medical college and Research Institute/Dr. D. Y. Patil University/Pune, Maharashtra, India \\ 3-Dr.D.Y.Patil Medical college and Research Institute/Dr. D. Y. Patil University/Pune, Maharashtra, India
}

Abstract: Background- Hypertension and Diabetes are known to have autonomic dysfunction and indices of heart rate variability provide window to understand the autonomic modulation of cardio respiratory systems.

Hypothesis- Co existing morbidity in Hypertensive diabetic group will cause enhancement of autonomic dysfunction.

Design- Hospital based case-control study. Hypertensive and Hypertensive diabetics (30 males each), not on regular medicines were selected from the Medicine and Diabetic OPD over a period of one year, and included in the study. Age and sex matched healthy controls were selected from hospital staff.Heart rate variability tests were done in the research laboratory of department of Physiology.

Materials and methods- After proforma the basal heart rate and resting blood pressures were recorded for each subject. Valsalva maneuver and the ECG recording was done on Polyrite-D Machine for one minute. Valsalva ratio was calculated.

Statistical analysis- ANOVA, Tuckeys, and Pearson co-relation coefficient tests were used to analyze the appropriate data.

Results- The mean VR of hypertensive subjects was.1.2647 \pm 0.1608 , statistically not significant when compared to healthy $(1.3137 \pm 0.1714)$. The mean VR of hypertensive diabetics was $1.1883 \pm 0.1302$,statistically highly significant.

Key words- valsalva ratio, HRV, autonomic functions, frequency analysis

\section{Introduction}

Diabetes mellitus( Type II) and hypertension are two of the most common diseases in Westernized, industrialized civilizations, and the frequency of both diseases increases with increasing age. ${ }^{1}$ Although diabetes mellitus is associated with a considerably increased cardiovascular risk, the presence of hypertension in the diabetic(type II) individual markedly increases morbidity and mortality

Several factors are known to regulate blood pressure. The nervous system helps to maintain blood pressure by adjusting the size of the blood vessels, and by influencing the heart pumping action. ${ }^{2}$ The autonomic nervous system plays crucial role in blood pressure (BP) and heart rate (HR) control and may thus be an important pathophysiological factor in the development of hypertension. It is also known that dysfunction of the autonomic nervous system is associated with increased risk of mortality in patients with diabetes, survivors of myocardial infarction and unselected middle-aged and elderly subjects. ${ }^{3}$

The association of autonomic imbalance with incident cardiovascular disease and all-cause mortality in the general population has been attributed to subclinical coronary artery disease, poor health and glucose intolerance. ${ }^{4}$ Subjects with diabetes(type II) are known to be at high risk of developing autonomic dysfunction, and autonomic dysfunction is already present in newly diagnosed diabetes(type II). Hypertension in the diabetic individual markedly increases the risk and accelerates the course of cardiac disease, peripheral vascular disease, stroke, retinopathy, and nephropathy. ${ }^{1}$ Our understanding of the factors that markedly increase the frequency of hypertension in the diabetic individual remains incomplete.

Heart rate variability is mainly a reflection of the influence of the autonomic nervous system on the sinus node of the heart. The heart rate alters with many of the changes in demand on the cardiovascular system that are related to changes in respiration, posture and physical or mental activity. These changes are invoked by the control mechanism that coordinate the total pattern of activity in the individual. Spectral analysis of HRV can be a powerful tool to assess ANS function. It is not only useful when studying the pathophysiological process in certain diseases but also may be used in daily clinical practice. ${ }^{4}$

The different types of autonomic function measures so far have not been compared in one single study population. ${ }^{3}$. Studies have identified abnormal HRV in systemic hypertension, The data examining the association between HRV and blood pressure in hypertensive and diabetic hypertensive patients is very small 
scale. ${ }^{5}$ This work was planned to study the role of autonomic function tests in hypertensive and hypertensive diabetic(type II) patients. The coexistence of the Hypertension and Type II Diabetes predicted to increase the autonomic dysfunction, and sympatho-vagal imbalance to be studied with the changes in the frequency parameters accordingly.

The present study was conducted in the Department of Physiology in collaboration with the Department of Medicine of Pad. Dr. D. Y. Patil Medical College, Pimpri, Pune,

90 male subjects in the age group of 40 to 65 years were selected for the study. 30 male subjects were patients of Hypertension and 30 male subjects were patients of Hypertension with Type II Diabetes Mellitus who attended the OPD of the Department of Medicine of Pad. Dr. D. Y. Patil Medical College, Pimpri, Pune, Maharashtra. 30 male healthy subjects of the same age group served as control..

\section{Settings and design}

Hospital based cross-sectional study

\section{Inclusion criteria}

Our study design included hypertensive and hypertensive diabetic(type II) subjects attending OPD and on treatment were tested for autonomic parameters. Age matched controls were selected simultaneously.

\section{Exclusion criteria-}

Subjects were excluded if they met any of the following criteria:

1) stroke.

2) Myocardial infarction or Congestive heart failure, atrial fibrillation,

3) Organ failure

4) Parkinson's disease

5) Juvenile Diabetes mellitus.

6) Morbidly obese subjects.

Thorough clinical examination of each subject was done and detailed history was taken with reference to duration of hypertension and diabetes(type II), family history, past history, personal history and drug history. General physical examination was done.

Height $(\mathrm{cm})$, weight $(\mathrm{kg})$ and blood pressure was measured. BMI was calculated from height and weight measurements. Blood pressure was recorded in supine position. Three readings were taken and average of second and third was used for the study. BP measurement was done by both auscultatory and palpatory method. Pulse rate, rhythm and character were assessed. All this information was recorded on a prepared questionnaire. Pre-test instructions were given to avoid consumption of any drugs (anti hypertensive and oral hypoglycemic drugs) that may alter the autonomic function 48 hours prior to the test. The patients were advised to have a silent sleep without medicine at the previous night. On the day of the test, no cigarette, nicotine, coffee, food or drugs orally or other routes were permitted for three hours prior to the test. The same procedures were also followed for the Healthy male subjects.

The tests were performed using Polyrite-D (RMS) machine in an equable environment with room temperature, in research laboratory room at the Department of Physiology of Pad. Dr. D. Y. Patil Medical College, Pimpri, Pune, Maharashtra. The lab is sound proof and air conditioned to provide comfort while performing tests.

The subjects were grouped as follows:

GROUP I- Healthy controls

GROUP II- Hypertensive patients

GROUP III-Hypertensive diabetic patients (Type II DM).

Heart rate response to Valsalva maneuver ${ }^{6,7,8,9,10}$

Apparatus: Polyrite - D, timer, Nose clip and modified mercurial sphygmomanometer. A mercurial manometer was improvised by modifying a mercurial sphygmomanometer and connected directly to the tube leading to mercury bulb.

Procedure:- The test was done after another 5 minutes interval of rest in sitting posture. The subject was instructed to exhale forcefully through the tube of the modified Mercurial sphygmomanometer and to maintain pressure in the manometer upto $40 \mathrm{~mm} \mathrm{Hg}$ for 15 seconds. ECG recording were taken during the maneuver and continued for about 30 seconds after the performance. The maneuver was repeated three times with few minutes time interval of rest. Nasal clip was used to stop nasal breathing of the subject during this maneuver. 
Calculation: The ratio of the longest RR interval after blowing to the shortest RR interval during blowing or immediately after was calculated. The highest ratio of the three maneuvers was used as a result of Valsalva ratio(VR).

Frequency domain analysis of heart rate variability-

Freuency domain analysis values for very low frequency (VLF), High frequency (HF),Low frequency (LF), and ratio of low frequency to high frequency (LF/HF) were recorded on Polyrite ECG recordings during Valsalva Maneuer for the duration of one minute.

\section{Statistical analysis-}

ANOVA, Tuckeys, and Pearson co-relation coefficient tests were used to analyze the appropriate data. ${ }^{12}$

\section{Observation and Results}

Table 1. gives the Autonomic function tests result of Valsalva ratio in all three groups.

\begin{tabular}{|l|l|l|l|}
\hline HRV Parameters & Group I & Group II & Group III \\
\hline VR & $1.3137 \pm 0.1714$ & $1.2647 \pm 0.1608$ & $1.1883 \pm 0.1302 *$ \\
\hline
\end{tabular}

Table 2. Comparision of HRV measures during Valsalva maneuver in GROUP I, II, III (Values are in Mean+Std.Dev.)

\begin{tabular}{|l|l|l|l|}
\hline HRV measures & Group I & Group II & Group III \\
\hline VLF & $1.747 \pm 6.149$ & $2.087 \pm 7.518$ & $3.153 \pm 5.336$ \\
\hline LF & $2.623 \pm 5.563$ & $2.983 \pm 5.976$ & $1.867 \pm 3.833$ \\
\hline HF & $0.8667 \pm 0.8612$ & $0.8655 \pm 1.518$ & $0.65 \pm 1.227$ \\
\hline LF/HF & $2.1 \pm 2.205$ & $3.363 \pm 2.444$ & $2.407 \pm 2.44$ \\
\hline
\end{tabular}

\section{Discussion}

The average ratio in group I was $1.3137 \pm 0.1714$. As compared to Group I in Group II, VR was reduced ( Average VR 1.2647 \pm 0.1608 ). This could be due to Parasympathetic dysfunction in Hypertension. ${ }^{13}$ Also in Group III VR was significantly reduced $1.1883 \pm 0.1302$ ). his may be because of sympathetic dominance and due to parasympathetic dysfunction. ${ }^{14}$.In Group III, VR was more reduced than in Group II. This suggestd that in both hypertension and hypertension with diabetes,co existence of disease may have resulted in sympathetic overactivity and parasympathetic dysfunction. ${ }^{15}$.This autonomic dysfunction was more in hypertension with diabetes because of coexisting autonomic neuropathy and was statistically significant. ${ }^{16}$ It is known that Sympathetic and parasympathetic dysfunctions occur differently in Diabetes and hypertension.

Table 2 Indicated average values of Heart rate variability tests, frequency parameters during Valsalva aneuver .

During valsalva maneuver in Group II as compared to Group I, VLF, LF, LF/HF increased indicating sympathetic over activity. Although statistically not significant. HF was decreased; may indicate parasympathetic dysfunction. ${ }^{17}$

In Group III as compared to Group I, LF, HF were decreased and VLF, LF/HF increased. It may indicated parasympathetic dysfunction and sympathetic over activity respectively.

In Group III as compared to Group II, LF, HF, LF/HF were decreased and VLF was increased. This indicated sympathetic over activity.

Frequency parameters although showed shift indicating sympatho vagal balance disturbance, they were not statistically significant. This could be because of small sample size , varied duration of illness. ${ }^{17}$

No strong correlation of VR with confounding factors such as BMI, SBP, DBP, but they were negatively correlated ${ }^{18}$

\section{Results and Conclusion:-}

- Changes in the valsalva maneuver occur due to changes in cardiac vagal efferents and sympathetic vasomotor activity which are stimulated by carotid sinus and aortic arch Baroreceptors and other intrathoracic stretch receptors.

- As VR decreases there is failure to decrease HR after strain which suggests Parasympathetic dysfunction.

- Autonomic dysfunction due to co-morbidity contributed highly significant variation compared to healthy subjects.

- Our study shows that low heart rate variability in hypertensives and hypertensive diabetics as compared to healthy subjects. 
- $\quad$ Lower HRV is associated with higher values of blood pressures putting subjects with such trends at a higher risk of developing hypertension.

\section{References}

[1] M Epstein and JR Sowers. Diabetes mellitus and hypertention. Hypertension. 1992;19:403-418.

[2] Pikkujämsä SM, Huikuri HV, Airaksinen KE, Rantala AO, Kauma H, Lilja M, Savolainen MJ, Kesäniemi YA. Heart rate variability and baroreflex sensitivity in hypertensive subjects with and without metabolic features of insulin resistance syndrome. Am J Hypertens. 1998 May;11(5):523-31.

[3] Jeanet Gerritsen, MSC ${ }^{1}$, Jacqueline M, Dekkar, PHD ${ }^{2}$, Ben J, TenVoorde, PHD ${ }^{1}$, Piet J. Kostense, PHD ${ }^{3}$, Robert J. Heine, MD ${ }^{2}$, Lex M. Bouter, PHD ${ }^{2}$, Rob M,PHD ${ }^{1}$ and Coen D.A. Stehouwer, MD ${ }^{2}$. Impaired Autonomic Function Is Associated With Increased Mortality, Hypertension or history of Cardiovascular Disease. Diabetes Care. October 2001 vol. 24 no. 10 1793-1798.

[4] Dr J. M. Karemaker, Heart Rate Variability:why do spectral analysis? 1996.

[5] 5) Singh JP, Larson MG, Tsuji H, Evans JC, O’Donnell CJ, Levy D. Reduced heart rate variability and new-onset hypertension: insights into pathogenesis of hypertension: the Framinggam Heart Study. Hypertension. 1998;32:293- 297.

[6] KASER, HAUSER, LONGO, JAMESON. Harrison's Principle of Internal Medicine(16 ${ }^{\text {th }}$. Edition)- volume 2, BRAUNWALD, FAUCI: Mc Graw-Hill-Medical Publishing Division,New Delhi, India: 28, 43, 44, 234, 422, 571, 1423, 2074, 2201, 2209, 2294, $2295,2428-2432$.

[7] Ewing DJ, Borsey DQ, Bellavere F and Clarke BF: Cardiac autonomic neuropathy in diabetes comparision of measures of RR interval; variation, Diabetologia, 1981;21:18-24.

[8] Hutchison's Clinical Method by Michael Swash( $25^{\text {th }}$ edition),ELBS-Great Britain:Page no. 326-327.

[9] 9)Ewing DJ; Analysis of heart rate variability and other non invasive tests with special reference to diabetes mellitus, Autonomic failure A Textbook of Clinical Disorders of the Autonomic Nervous Ststem, Banniste R and Mathias CJ: oxford University Press inc, New York, 1992; ( $3^{\text {rd }}$ edition), Page no. 312-333.

[10] Autonomic Function Testing- Clinical and examples by Alejandro Ortiz-Burgos, M.D-University of Miami, Internal Medicine, $27^{\text {th }}$ June 2006.

[11] Antti Ylitalo, Cardiovascular autonomic regulation in systemic hypertension, Oulu 1999.

[12] 12) Park's Text book of Preventive and Social Medicine, by Dr. K. Park-20 ${ }^{\text {th }}$ edition, Commercial Law Publishers, New Delhi, India:559-560.

[13] Piccricillo G, et al, Heart rate variability in hypertensive subjects. Int J Cardiol. 1996;53(3):291-8

[14] Naohiko Takahashi, Mikiko Nakagawa, Tetsunori Saikawa,, Tatsuhiko Ooie, Kunio Yufu, Sakuji Shigematsu, Masahide Hara, Hiroshi Sakino, Isao Katsuragi, Toshimitsu Okeda, Hironobu Yoshimatsu, Toshiie Sakata, Effect of Essential Hypertension on Cardiac Autonomic Function in Type 2 Diabetic Patients. Vol. 38 No. 12001

[15] KASER, HAUSER, LONGO, JAMESON. Harrison's Principle of Internal Medicine(16 ${ }^{\text {th }}$. Edition)- volume 2 , BRAUNWALD, FAUCI: Mc Graw-Hill-Medical Publishing Division,New Delhi, India: 28, 43, 44, 234, 422, 571, 1423, 2074, 2201, 2209, 2294, 2295, 2428-2432

[16] Peter Kempler et al, Relationship between hypertension, autonomic neuropathy and the cardiovascular reflex tests in type 2 diabetes. 2009

[17] Robert J, Eleanor M, Stephen G, John B, Alan F, David A, Impact of Type 2 Diabetes Mellitus on Sympathetic Neural Mechanisms in Hypertension. Circulation. 2003;108:3097-3101.

[18] Julian F, Shelby S, Jos F, The relationship of autonomic imbalance, heart rate variability and cardiovascular disease risk factors. Cardiology 2009;9:543. 